\title{
Hepatic Arterial Infusion Chemotherapy Combined with Radiation Therapy for Advanced Hepatocellular Carcinoma with Tumor Thrombosis of the Main Trunk or Bilobar of the Portal Vein
}

\author{
Yumi Kosaka ${ }^{\text {a }}$ Tomoki Kimura ${ }^{c}$ Tomokazu Kawaoka ${ }^{a}$ Yutaro Ogawa ${ }^{a}$ \\ Kei Amioka ${ }^{a}$ Kensuke Naruto ${ }^{a}$ Yuki Yoshikawa ${ }^{a}$ Chihiro Kikukawa ${ }^{a}$ \\ Yosuke Suehiro $^{a}$ Kenji Yamaoka ${ }^{a}$ Yuwa Ando $^{a}$ Shinsuke Uchikawa ${ }^{a}$ \\ Kei Morio ${ }^{a}$ Takashi Nakahara ${ }^{a}$ Eisuke Murakami ${ }^{a}$ Shoichi Takahashi ${ }^{a}$ \\ Masataka Tsuge $^{a} \quad$ Akira Hiramatsu $^{a}$ Michio Imamura $^{a}$ Keigo Chosa ${ }^{b}$

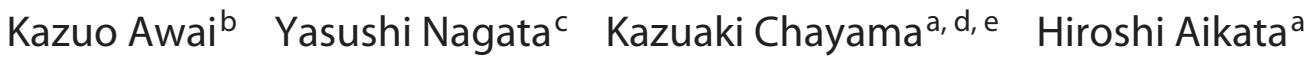

aDepartment of Gastroenterology and Metabolism, Hiroshima University, Hiroshima, Japan; ${ }^{\mathrm{b}}$ Diagnostic Radiology, and, Hiroshima University, Hiroshima, Japan; 'Therapeutic Radiology, Institute and Graduate School of Biomedical Science, and, Hiroshima University, Hiroshima, Japan; 'Research Center for Hepatology and Gastroenterology, Hiroshima University, Hiroshima, Japan; eInstitute of Physical and Chemical Research (RIKEN) Center for Integrative Medical Sciences, Yokohama, Japan

\section{Keywords}

Hepatocellular carcinoma · Vp4 · Hepatic arterial infusion chemotherapy · Radiation therapy · Treatment response

\begin{abstract}
Background: Overall survival of patients with advanced hepatocellular carcinoma (HCC) with Vp4 (tumor thrombosis of the main trunk or bilobar of the portal vein) is extremely poor. Purpose: The purpose of this study is to clarify the prognosis of hepatic arterial infusion chemotherapy (HAIC) combined with radiation therapy (RT) for advanced HCC with Vp4 and to analyze the factors that contribute to the prognosis. Methods: In this retrospective cohort study, 51 HCC patients who were treated with HAIC and RT for portal vein tumor thrombosis and met the following criteria were
\end{abstract}

karger@karger.com www.karger.com/lic

Karger $\frac{1}{\%}$

GOPEN ACCESS
(C) 2021 The Author(s)

Published by S. Karger AG, Basel

This article is licensed under the Creative Commons AttributionNonCommercial-NoDerivatives 4.0 International License (CC BY NC-ND) (http://www.karger.com/Services/OpenAccessLicense). Usage and distribution for commercial purposes as well as any distribution of modified material requires written permission. enrolled: (i) with Vp4; (ii) Child-Pugh score of 5-7; (iii) Eastern Cooperative Oncology Group performance status of 0 or 1; (iv) no history of systemic therapy; and (v) from September 2004 to April 2019. Results: Median overall survival and median progression-free survival were 12.1 and 4.2 months, respectively. Multivariate analysis showed $>50 \%$ of relative tumor volume in the liver (HR, 3.027; $p=0.008)$ and extrahepatic spread with (HR, 3.773; $p=0.040)$ as significant and independent factors of OS. The total overall response rate (ORR) was 19.6\%; ORR in main tumor was $13.7 \%$; and ORR in Vp4 was $51.0 \%$. None of the patients who received HAIC combined with RT for advanced HCC with Vp4 developed hepatic failure. This combination therapy of HAIC with RT was safe and well tolerated in all cases. Conclusion: Combination therapies of HAIC and RT might be good therapy for advanced HCC with Vp4.

(c) 2021 The Author(s)

Published by S. Karger AG, Basel 


\section{Introduction}

Hepatocellular carcinoma (HCC) is a leading cause of cancer-related death worldwide, and the prognosis of unresectable HCC is extremely poor $[1,2]$. HCC occurs frequently in patients with chronic hepatitis or liver cirrhosis due to either hepatitis B or C viral infection, alcohol use, nonalcoholic steatohepatitis or diabetes [3]. The survival of patients with HCC has gradually improved following the development of new diagnostic techniques and advancements in therapeutic modalities, such as surgical resection, radiofrequency ablation, percutaneous ethanol injection, transcatheter arterial chemoembolization (TACE), radiation therapy (RT), hepatic arterial infusion chemotherapy (HAIC) and multiple molecular targeted agents (MTAs) [3-19]. However, the prognosis of patients with advanced HCC, especially those with advanced portal vein tumor thrombosis (PVTT), remains poor [20].

The recently developed tyrosine kinase inhibitors (TKIs) such as sorafenib and lenvatinib have been approved as first-line systemic therapies for unresectable advanced HCC. In addition, regorafenib, ramucirumab, and cabozantinib have been confirmed to be effective as second-line systemic therapies for unresectable advanced HCC. These MTAs have been reported to improve the prognosis of advanced HCC.

However, HCC patients with massive PVTT often have portal hypertension, a risk of esophagogastric varix rupture and a poor prognosis due to liver dysfunction [3]. These unfavorable conditions often lead to the use of insufficient doses of MTAs. In particular, patients with HCC with tumor thrombosis of the main trunk or bilobar of the portal vein ( $\mathrm{Vp} 4)$ have a very poor prognosis. In addition, HCC with Vp4 has often been an exclusion criterion in previous pivotal trials; thus, the prognosis of these patients is unclear.

On the other hand, there are many reports that HAIC is an effective treatment for HCC with PVTT $[8,9,21]$. In retrospective cohort studies comparing HAIC and sorafenib, HAIC was the more effective treatment for HCC with PVTT $[22,23]$. HAIC includes either an implantable drug delivery system, which delivers repeated arterial infusions of the chemotherapeutic agents, or 1-shot arterial infusion of cisplatin (CDDP) [24]. The phase 3 SILIUS trial was conducted as a prospective study comparing sorafenib plus HAIC using low-dose FP versus sorafenib alone in patients with advanced HCC [25]. That trial failed to demonstrate the efficacy of HAIC. However, a subgroup analysis in the SILIUS trial of pa- tients with Vp4 showed that those treated with HAIC combined with sorafenib had a better prognosis compared with those treated with sorafenib alone. It was suggested that HAIC may provide a survival benefit for patients with advanced HCC with PVTT. Ikeda et al. [26] reported that sorafenib plus HAIC using CDDP resulted in a better prognosis compared with sorafenib alone. As that was a phase II trial, HAIC has yet to be established as a standard treatment for advanced HCC, but HAIC is considered an effective treatment for advanced HCC with PVTT.

Advances in 3D-CRT have enabled reliable irradiation of tumors with minimal radiation delivered to normal tissues, resulting in an enhanced antitumor effect and reduced damage in normal tissues. This modality renders local irradiation of PVTT possible [7, 27]. In addition, there are some reports of a good response rate after combination therapy of HAIC and RT in patients with advanced HCC and PVTT [3, 7, 27-29].

However, those reports involved advanced HCC with PVTT ranging from Vp1 to Vp4, with no studies conducted on $\mathrm{Vp} 4$ cases alone; thus, there is no established treatment for advanced HCC with Vp4. Here, we analyzed the effectiveness and safety of HAIC combined with RT for advanced HCC with Vp4.

\section{Materials and Methods}

\section{Patients}

In this retrospective cohort study, HCC patients treated with HAIC and RT for PVTT who met the following criteria were enrolled: (i) presence of Vp4; (ii) Child-Pugh score of 5-7; (iii) ECOG PS of 0 or 1; (iv) no history of systemic therapy; and (v) from September 2004 to April 2019. This study was approved by the Institutional Review Board of Hiroshima University Hospital based on the ethical principles of the Declaration of Helsinki. Detailed written informed consent was obtained from each patient for each treatment performed.

\section{Therapeutic Protocol}

Hepatic Arterial Infusion Chemotherapy

In this study, 2 HAIC regimens were used: continuous infusion based on the reservoir system (continuous HAIC) and transcatheter arterial infusion chemotherapy via bolus injection (singledose HAIC). In Japan, continuous HAIC combination of low-dose CDDP and intra-arterial 5-fluorouracil (5-FU) combined with interferon and single-dose HAIC (1-shot intra-arterial infusion of CDDP) are commonly used as HAIC regimens, and their effectiveness has been reported previously [19-25]. The duration of each course of continuous HAIC was 2 weeks. In the continuous HAIC regimen, 5 -FU was administered at a dose of $330 \mathrm{mg} / \mathrm{m}^{2}$ per day on days $1-5$ of weeks 1 and 2 using a mechanical infusion pump for $24 \mathrm{~h}$. CDDP was injected intra-arterially at a dose of $20 \mathrm{mg} / \mathrm{m}^{2}$ 
per day on days 1-5. Interferon was given intramuscularly on days 1,3 , and 5 of each week. There was a 2 - to 4 -week interval of no drug administration after each course of treatment. In the singledose HAIC regimen, patients were administered a CDDP dose of $65 \mathrm{mg} / \mathrm{m}^{2}$ (maximum dose, $100 \mathrm{mg} / \mathrm{m}^{2}$ ) through a catheter into the whole liver at a rate of $2 \mathrm{~mL} / \mathrm{min}$. The dose was decreased by $75 \%$ when the estimated glomerular filtration rate was $<60 \mathrm{mg} /$ $\mathrm{min} / 1.73 \mathrm{~m}^{2}$. When CDDP was infused to a part of the liver, the dose was adjusted roughly by the proportion of the tumor volume in the liver based on computed tomography (CT) images. To prevent CDDP-induced renal dysfunction, adequate hydration was ensured before and after drug administration. This treatment was repeated at an interval of 4-6 weeks. In principle, HAIC was repeated several times during the treatment until we considered it impossible for patients to continue HAIC based on the following criteria: (i) a change in the ECOG PS to 3 or 4; (ii) adverse events of grade 4 according to the Common Technology Criteria for Adverse Events (CTCAE) v4.0; or (iii) request of treatment termination by the patient.

\section{Radiation Therapy}

All patients received 3D-CRT concomitantly with the first course of HAIC. 3D-CRT consisted of high-energy photon beam irradiation using 18,10 , or $6 \mathrm{MV}$, delivered by a $3 \mathrm{D}$ conformal technique (CLINAC $2300 \mathrm{C} / \mathrm{D}$ or CLINAC iX linear accelerator; Varian Medical Systems Inc., Palo Alto, CA, USA) in the Division of Radiation Oncology at our hospital. Planned CT was used to determine the total tumor volume confined to the PVTT. The clinical target volume was established as the gross tumor volume plus the intrahepatic tumor volume in the basal portion of the PVTT. The planning target volume comprised the clinical target volume plus a 10- to 20-mm margin in all directions to account for internal motion and setup errors. Four to 5 portal fields were used. Data regarding the outlined target volume, total liver tissue, and at-risk structures, including the spinal cord, both kidneys, and nearby intestinal tract targets, were transferred to the treatment planning system (Pinnacle 3; Philips Medical Systems, Eindhoven, The Netherlands) with reference to the diagnostic contrast-enhanced CT images. The irradiation dose was 30,39 , or $45 \mathrm{~Gy}$, depending on the normal tissue volume and liver function; $95 \%$ of the planning target volume received at least $95 \%$ of the irradiation dose.

\section{Assessment of Treatment Efficacy}

The treatment response was assessed by contrast-enhanced CT at 4 weeks after completion of each treatment course and then every 2-3 months. The response was defined according to the modified Response Evaluation Criteria in Solid Tumors (mRECIST) [30]. According to mRECIST, the therapeutic effect was judged by measuring the longest diameters and the degree of contrast of tumor thrombosis.

\section{Evaluation of Treatment-Related Adverse Events}

The safety assessment included documentation of adverse drug reactions, clinical laboratory tests, physical examinations, and measurements of vital signs. Adverse drug reactions were defined according to the CTCAE v4.0 (http://ctep.cancer.gov/protocolDevelopment/electronic_applications/docs/ctcaev3.pdf) [31]. Radiation-induced liver disease (RILD) is divided into "classic" and "non-classic" RILD. Classic RILD, which usually occurs between 2 weeks and 3 months after treatment, involves anicteric hepato- megaly and ascites, and elevation of the alkaline phosphatase level to at least 2-fold the upper limit of normal or the pretreatment value in the absence of tumor progression. Classic RILD can occur in patients with good liver function. Non-classic RILD, which usually occurs between 1 week and 3 months after treatment, involves elevation of the alkaline phosphatase level to $>5$-fold the upper limit of normal and CTCAE grade 4 in patients with a baseline value $>5$-fold the upper limit of normal within 3 months after completion of RT, or a decrease in liver function (defined by an increase in the Child-Pugh score of $>2$ points), in the absence of classic RILD. The end point was described in patients with poor liver function.

\section{Treatments after Combination Therapy of HAIC and RT}

If the volume of intrahepatic tumor or PVTT decreased (i.e., downstaging) after determining the therapeutic effect, patients with good liver function and a PS of 0 or 1 received hepatic resection as conversion therapy. Patients who attained a partial response (PR) subsequently received repeated HAIC. Patients with stable disease (SD) or progressive disease (PD) who had good liver function and a PS of 0 or 1 received TKIs or TACE. Patients with a complete response (CR) were observed during the clinical course periodically without additional therapy. The patient who discontinued treatment was designated as dropout (DO), and there was only 1 patient.

\section{Statistical Analysis}

Statistical analysis was performed using the Mann-Whitney U test, logistic regression analysis, and $\chi^{2}$ test as appropriate. We evaluated OS and progression-free survival (PFS). The cumulative survival rate was calculated from the initial date of therapy. PFS was calculated from the date of PD confirmation. These parameters were assessed using the Kaplan-Meier life table method, and differences in survival were evaluated by the log-rank test. Multivariate analysis of predictors of OS were assessed by Cox proportional hazards regression. $p<0.05$ was considered statistically significant. All analyses were performed using SPSS software.

\section{Results}

\section{Patient Background Characteristics}

Fifty-one patients were enrolled in this retrospective cohort study. The characteristics of the patients and the clinical data at the time of the initial treatment are summarized in Table 1 . The median age of the patients was 65 years (range, 30-86 years), and 48 patients were male. Child-Pugh scores of 5, 6, and 7 were noted in 20 (39.2\%), $25(49.0 \%)$, and $6(11.8 \%)$ patients, respectively. Regarding the etiology, 13 and 20 patients were positive for the HBs antigen and hepatitis $\mathrm{C}$ virus antibodies, respectively. The relative tumor volume in the liver was $<50 \%$ in 38 (74.5\%) patients. The median size of the liver tumors was $70 \mathrm{~mm}$ (range, 25-170 mm). HCC with tumor thrombosis of the main trunk or bilobar of the portal vein was present in 47 (92.2\%) and in 27 (52.9\%) patients, respec- 


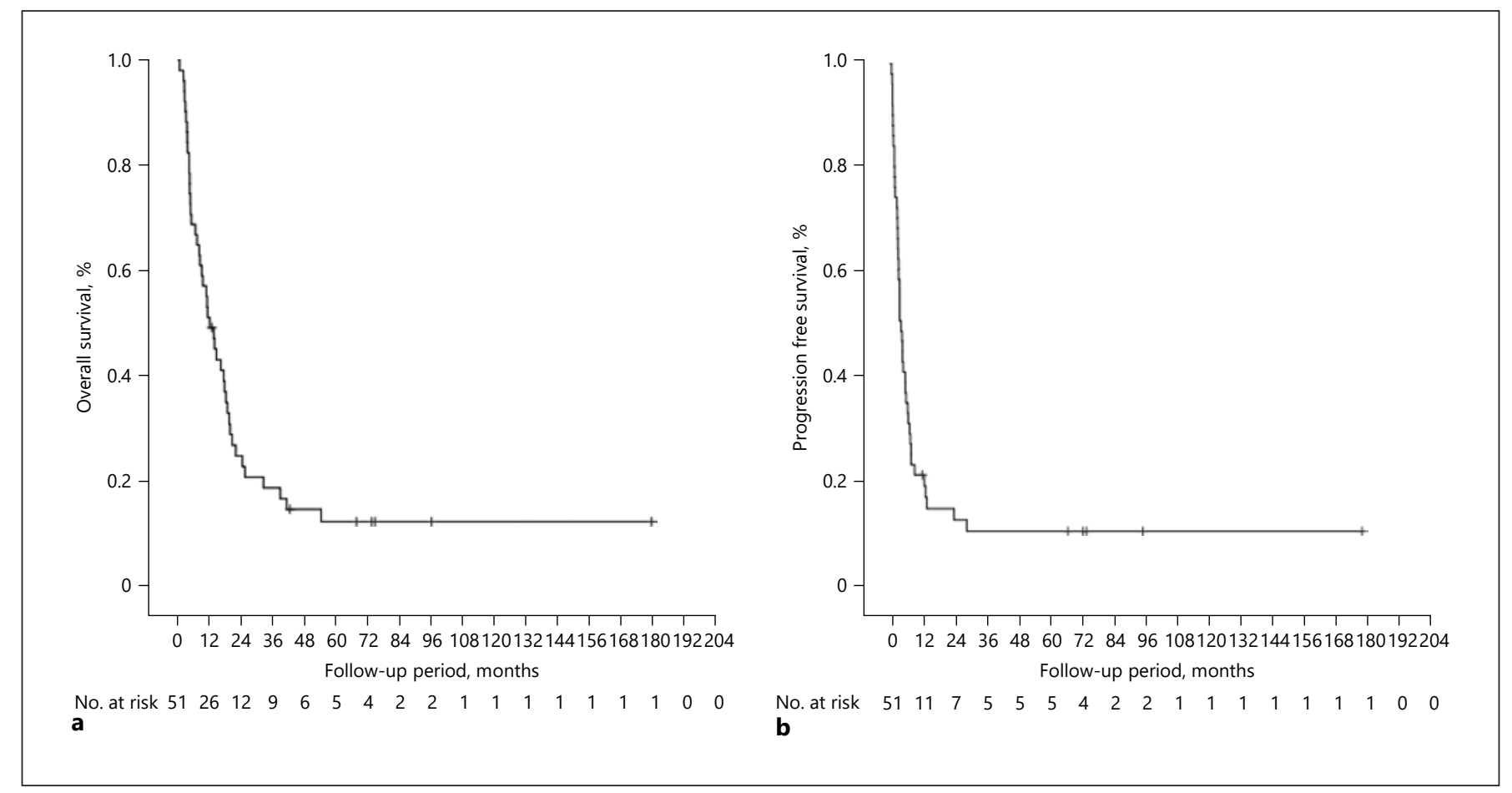

Fig. 1. Overall survival and PFS of all patients. a Overall survival of all patients. b PFS of all patients. PFS, progression-free survival.

Table 1. Baseline characteristics of the 51 study patients

\begin{tabular}{lc}
\hline Characteristic & $\begin{array}{c}\text { Median (range) } \\
\text { or patients, } n\end{array}$ \\
\hline Age, years & $65(30-86)$ \\
Sex (M/F) & $48 / 3$ \\
Etiology (HBV/HCV/HBV + HCV/other) & $13 / 20 / 2 / 16$ \\
Total bilirubin, mg/dL & $0.9(0.4-1.8)$ \\
Albumin, g/dL & $3.8(2.8-5.0)$ \\
Prothrombin consumption test, $n(\%)$ & $82(58-118)$ \\
Child-Pugh score $(5 / 6 / 7)$ & $20 / 25 / 6$ \\
Relative tumor volume in the liver $(<50 \% / \geq 50 \%)$ & $38 / 13$ \\
Liver tumor size, mm & $70(25-170)$ \\
Vv (0/1/2/3) & $46 / 1 / 1 / 3$ \\
Intrahepatic PVTT (bilobar/unilobar) & $27 / 24$ \\
Main portal trunk tumor thrombus $($ with/without) & $47 / 4$ \\
Occupancy rate of main portal trunk $(<50 \% / \geq 50 \%)$ & $38 / 13$ \\
Extrahepatic spread $($ without/with) & $46 / 5$ \\
HCC stage (III/IVa/IVb) & $11 / 36 / 4$ \\
AFP, ng/mL & $227.9(2.6-529,500)$ \\
\end{tabular}

AFP, alpha-fetoprotein; HBV, hepatitis B virus; HCC, hepatocellular carcinoma; HCV, hepatitis $\mathrm{C}$ virus; $\mathrm{Vv}$, venous invasion; PVTT, portal vein tumor thrombosis. ${ }^{\text {a According }}$ to the Liver Cancer Group of Japan. 


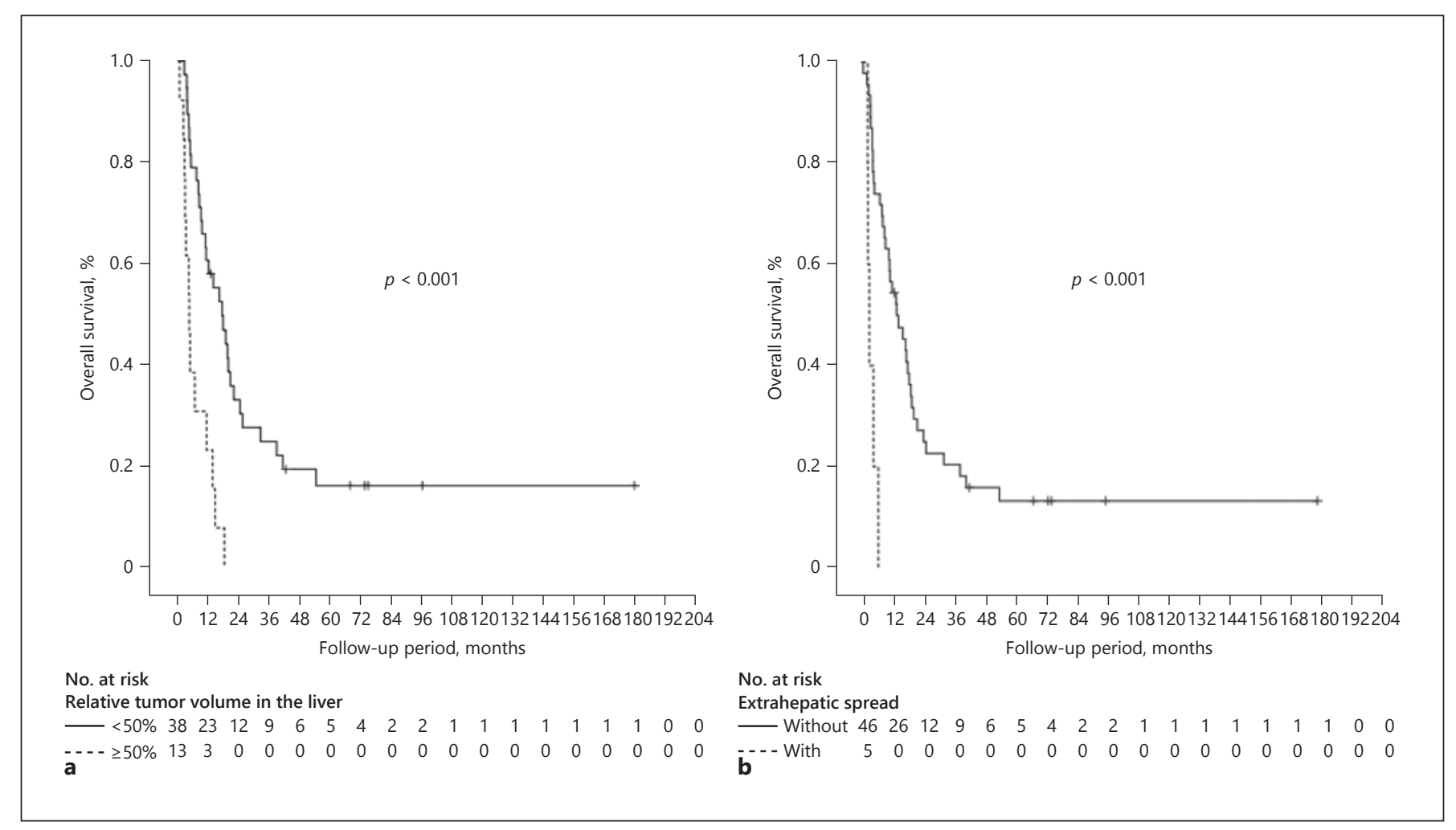

Fig. 2. Comparison of overall survival according to the relative tumor volume in the liver and extrahepatic. a Comparison of overall survival according to the relative tumor volume in the liver $(<50 \%$ vs. $>50 \%)$. b Comparison of overall survival between patients with and those without extrahepatic spread.

tively, with some of these patients having both. Five $(9.8 \%)$ patients had extrahepatic metastases. The median AFP level was $227.9 \mathrm{ng} / \mathrm{mL}$ (range, 2.6-529,500 ng/mL).

\section{OS and Factors Affecting OS}

The median OS was 12.1 months in all patients (Fig. 1a). Univariate analyses identified a main tumor diameter $>70 \mathrm{~mm}(p=0.049)$, relative tumor volume in the liver $>50 \%(p<0.001)$, and extrahepatic spread $(p<0.001)$ as significant factors predicting unfavorable OS. In the multivariate analysis, a relative tumor volume in the liver $>50 \%$ (hazard ratio [HR], 3.027; 95\% CI, 1.336-6.861; $p=0.008)$ and extrahepatic spread (HR, 3.773; 95\% CI, $1.006-13.360 ; p=0.040)$ remained significant and independent factors predicting poor OS (Fig. 2a, b; Table 2).

\section{PFS and Factors Affecting PFS}

The median PFS was 4.2 months (Fig. 1b). Univariate analyses identified a main tumor diameter $>70 \mathrm{~mm}(p=$ $0.009)$, relative tumor volume in the liver $>50 \%$ ( $p=$ $0.018)$, and extrahepatic spread $(p=0.018)$ as significant predictors of poor PFS. In the multivariate analysis, extrahepatic spread (HR, 2.838; 95\% CI, 0.957-8.416; $p=$ 0.060 ) remained a significant and independent factor predicting unfavorable PFS (Table 3).

\section{Overall Response Rate to HAIC Combined with RT}

Based on the mRECIST, the total overall response rate (ORR) was $19.6 \%$. The ORRs of the main tumor and PVTT were 13.7 and 51.0\%, respectively. The overall CR, PR, SD, and PD rates were 2.0, 17.6, 50.9, and 27.5\%, respectively (Table 4). The CR, PR, SD, and PD rates of the main tumor were $2.0,11.7,56.8$, and $27.5 \%$, respectively, and those of the PVTT were 2.0, 49.0, 29.4, and 17.6\%, respectively.

\section{OS According to the Treatment Response}

OS according to the overall treatment response is shown in Figure 3a. The median OS of responders (i.e., those with a CR or PR), of the patients with $\mathrm{SD}$ and $\mathrm{PD} /$ DO were $19.8,14.6$, and 4.2 months, respectively. 
Table 2. Univariate and multivariate analyses of unfavorable factors predicting overall survival after HAIC and RT

\begin{tabular}{|c|c|c|c|c|}
\hline \multirow[t]{2}{*}{ Parameter } & \multirow{2}{*}{$\begin{array}{l}\text { Univariate analysis } \\
p \text { value }\end{array}$} & \multicolumn{3}{|c|}{ Multivariate analysis } \\
\hline & & HR & $95 \% \mathrm{CI}$ & $p$ value \\
\hline Age $(\geq 65 /<65$ years $)$ & 0.632 & & & \\
\hline Child-Pugh score $(7 / 5-6)$ & 0.243 & & & \\
\hline Diameter of main tumor $(\geq 70 /<70 \mathrm{~mm})$ & 0.049 & & & \\
\hline Relative tumor volume in the liver $(\geq 50 \% /<50 \%)$ & $<0.001$ & 3.027 & $1.336-6.861$ & 0.008 \\
\hline Intrahepatic PVTT (bilobar/unilobar) & 0.221 & & & \\
\hline Main portal trunk tumor thrombus (with/without) & 0.378 & & & \\
\hline Occupancy rate of main portal trunk $(\geq 50 \% /<50 \%)$ & 0.205 & & & \\
\hline Extrahepatic spread (with/without) & $<0.001$ & 3.773 & $1.006-13.360$ & 0.040 \\
\hline $\operatorname{AFP}(\geq 300 /<300 \mathrm{ng} / \mathrm{mL})$ & 0.305 & & & \\
\hline
\end{tabular}

HAIC, hepatic arterial infusion chemotherapy; RT, radiation therapy; PVTT, portal vein tumor thrombus; AFP, alpha-fetoprotein. According to the Liver Cancer Group of Japan.

Table 3. Univariate and multivariate analyses of unfavorable factors predicting PFS after HAIC and RT

\begin{tabular}{|c|c|c|c|c|}
\hline \multirow[t]{2}{*}{ Parameter } & \multirow{2}{*}{$\begin{array}{l}\text { Univariate analysis } \\
p \text { value }\end{array}$} & \multicolumn{3}{|c|}{ Multivariate analysis } \\
\hline & & HR & $95 \% \mathrm{CI}$ & $p$ value \\
\hline Age $(\geq 65 /<65$ years $)$ & 0.278 & & & \\
\hline Child-Pugh score $(7 / 5-6)$ & 0.341 & & & \\
\hline Diameter of main tumor $(\geq 70 /<70 \mathrm{~mm})$ & 0.009 & 1.982 & $1.026-3.827$ & 0.042 \\
\hline Relative tumor volume in the liver $(\geq 50 \% /<50 \%)$ & 0.018 & & & \\
\hline Intrahepatic PVTT (bilobar/unilobar) & 0.343 & & & \\
\hline Main portal trunk tumor thrombus (with/without) & 0.760 & & & \\
\hline Occupancy rate of main portal trunk $(\geq 50 \% /<50 \%)$ & 0.295 & & & \\
\hline Extrahepatic spread (with/without) & 0.018 & 2.838 & $0.957-8.416$ & 0.060 \\
\hline $\operatorname{AFP}(\geq 300 /<300 \mathrm{ng} / \mathrm{mL})$ & 0.326 & & & \\
\hline
\end{tabular}

PFS, progression-free survival; HAIC, hepatic arterial infusion chemotherapy; RT, radiation therapy; PVTT, portal vein tumor thrombus; AFP, alpha-fetoprotein. According to the Liver Cancer Group of Japan.

OS according to treatment response in the main tumor is shown in Figure 3b. The median OS of the responders was not reached and that of the patients with SD and $\mathrm{PD} /$ DO were 14.6 and 4.2 months, respectively.

OS according to the treatment response in the PVTT is shown in Figure 3c. The median OS of the responders, patients with SD and PD/DO were 19.4, 14.6, and 4.2 months, respectively. OS in all cases was stratified.

\section{Evaluation of Treatment-Related Adverse Events}

Treatment-related toxicities were observed in 44 $(86.3 \%)$ patients. The common adverse events were nausea, anorexia, general fatigue, and fever most of which were CTCAE grade 1 or 2 . The CTCAE grade 3 or 4 adverse events observed were an increased alanine amino-
Table 4. Objective response rate to combination HAIC and RT

\begin{tabular}{lccc}
\hline & $\begin{array}{l}\text { Total } \\
(n=51)\end{array}$ & $\begin{array}{l}\text { Main tumor } \\
(n=51)\end{array}$ & $\begin{array}{l}\text { PVTT } \\
(n=51)\end{array}$ \\
\hline $\mathrm{CR}, \%(n)$ & $2.0(1)$ & $2.0(1)$ & $2.0(1)$ \\
$\mathrm{PR}, \%(n)$ & $17.6(9)$ & $11.7(6)$ & $49.0(25)$ \\
$\mathrm{SD}, \%(n)$ & $50.9(26)$ & $56.8(29)$ & $29.4(15)$ \\
$\mathrm{PD}, \%(n)$ & $27.5(14)$ & $27.5(14)$ & $17.6(9)$ \\
$\mathrm{DO}, \%(n)$ & $2.0(1)$ & $2.0(1)$ & $2.0(1)$ \\
ORR, \% & 19.6 & 13.7 & 51.0 \\
DCR, \% & 70.5 & 70.5 & 80.4 \\
\hline
\end{tabular}

mRECIST, modified Response Evaluation Criteria in Solid Tumors; CR, complete response; $\mathrm{PR}$, partial response; $\mathrm{SD}$, stable disease; $\mathrm{PD}$, progressive disease; $\mathrm{DO}$, dropout; ORR, objective response rate; DCR, disease control rate. 

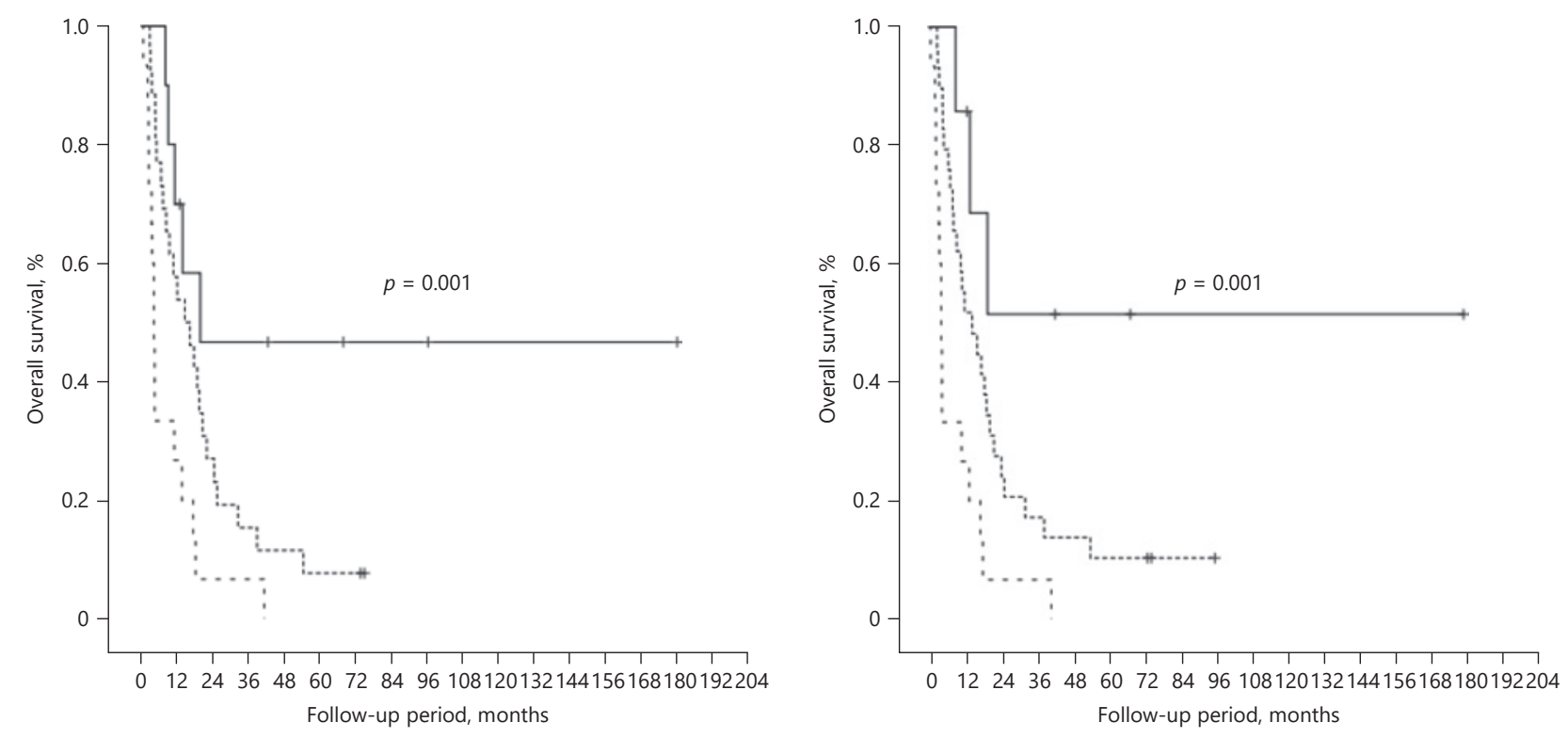

No. at risk

$\begin{array}{lllllllllllllllllll}-\mathrm{CR} / \mathrm{PR} & 10 & 7 & 4 & 4 & 3 & 3 & 2 & 2 & 2 & 1 & 1 & 1 & 1 & 1 & 1 & 1 & 0 & 0\end{array}$ ..... SD $\quad 26 \begin{array}{lllllllllllllllll}15 & 7 & 4 & 3 & 2 & 2 & 0 & 0 & 0 & 0 & 0 & 0 & 0 & 0 & 0 & 0 & 0\end{array}$

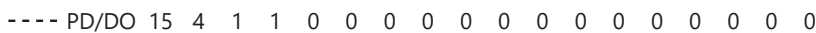
a

No. at risk

$\begin{array}{llllllllllllllllllll}\mathrm{CR} / \mathrm{PR} & 7 & 6 & 3 & 3 & 2 & 2 & 1 & 1 & 1 & 1 & 1 & 1 & 1 & 1 & 1 & 1 & 0 & 0\end{array}$ $\mathrm{SD} \quad 29 \begin{array}{lllllllllllllllll}16 & 8 & 5 & 4 & 3 & 3 & 1 & 1 & 0 & 0 & 0 & 0 & 0 & 0 & 0 & 0 & 0\end{array}$ b

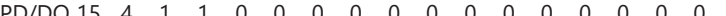

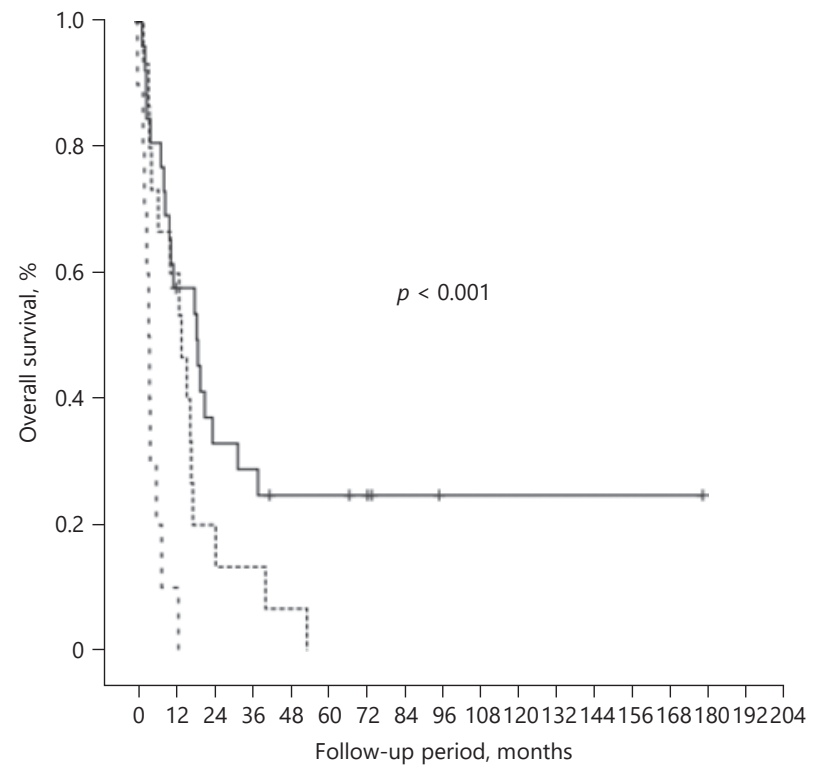

No. at risk

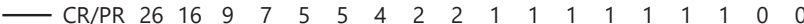

...... SD $\quad \begin{array}{llllllllllllllllll}15 & 9 & 3 & 2 & 1 & 0 & 0 & 0 & 0 & 0 & 0 & 0 & 0 & 0 & 0 & 0 & 0 & 0\end{array}$

c - - PD/DO $10 \begin{array}{lllllllllllllllll}10 & 0 & 0 & 0 & 0 & 0 & 0 & 0 & 0 & 0 & 0 & 0 & 0 & 0 & 0 & 0 & 0\end{array}$

Fig. 3. Comparison of overall survival between patients with assessment of treatment efficacy in main tumor and PVTT. a Comparison of overall survival among patients with a CR/PR, SD, and PD/DO in both the main tumor and PVTT. $\mathbf{b}$ Comparison of overall survival among patients with a CR/PR, SD, and PD/DO in the main tumor. c Comparison of overall survival among patients with a CR/PR, SD and PD/DO in the PVTT. PVTT, portal vein tumor thrombosis; CR, complete response; $\mathrm{PR}$, partial response; $\mathrm{SD}$, stable disease; $\mathrm{PD}$, progressive disease; $\mathrm{DO}$, dropout. 
Table 5. Treatment-emergent adverse events of HAIC and RT

\begin{tabular}{lcc}
\hline & $\begin{array}{l}\text { Any grade, } \\
\%(n)\end{array}$ & $\begin{array}{c}\text { Grade } 3 \text { or } \\
\text { higher, \% }(n)\end{array}$ \\
\hline Appetite loss & $21.6(11)$ & $11.8(6)$ \\
General fatigue & $19.6(10)$ & $9.8(5)$ \\
Aspartate aminotransferase increased & $11.8(6)$ & $7.8(4)$ \\
Alanine aminotransferase increased & $11.8(6)$ & $7.8(4)$ \\
Reservoir infection & $7.8(4)$ & $3.9(2)$ \\
Fever & $7.8(4)$ & 0 \\
Anemia & $5.9(3)$ & 0 \\
Cr increased & $5.9(3)$ & $5.9(3)$ \\
Neutropenia & $3.9(2)$ & 0 \\
Thrombocytopenia & $3.9(2)$ & 0 \\
Diarrhea & $3.9(2)$ & $1.9(1)$ \\
Vasculitis & $3.9(2)$ & $3.9(2)$ \\
Epigastralgia & $3.9(2)$ & $3.9(2)$ \\
Hepatic encephalopathy & $1.9(1)$ & $1.9(1)$ \\
\hline
\end{tabular}

HAIC, hepatic arterial infusion chemotherapy; RT, radiation therapy.

transferase/aspartate aminotransferase level $(n=8$, $15.6 \%)$, increased Cr level $(n=3,5.9 \%)$, vasculitis $(n=2$, $3.9 \%)$, and hepatic encephalopathy $(n=1,1.9 \%)$ (Table 5). Among the patients treated with the 5-FU-based regimen, port infection occurred in 4 patients, 2 of whom discontinued the regimen. None of the patients who received HAIC and RT for advanced HCC with Vp4 developed hepatic failure that met the criteria for classic or non-classic RILD.

\section{Treatments after Combination HAIC and RT}

Six $(11.8 \%)$ patients were downstaged after combination therapy of HAIC and RT and received hepatic resection as conversion therapy. Eleven $(21.6 \%)$ patients were treated with TACE. Twelve $(23.5 \%)$ patients were treated with the TKIs sorafenib $(n=8)$ and lenvatinib $(n=4)$.

\section{Discussion}

In this study, we analyzed the efficacy and clinical outcomes of combination HAIC and RT for advanced HCC with Vp4. The median OS and PFS of the study patients were 12.1 and 4.2 months, respectively. The total ORR was $19.6 \%$, but the ORR of the PVTT was $51.0 \%$. The combination of HAIC and RT for HCC with $\mathrm{Vp} 4$ yielded a favorable OS, and the resulting adverse events were relatively well tolerated.
Recently, it was reported that atezolizumab and bevacizumab combination therapy results in significantly better prognosis than does sorafenib in HCC patients. The criteria for clinical trials of atezolizumab and bevacizumab includes the patients with advanced HCC with Vp4, but no subgroup analysis has been done [32]. Therefore, it is necessary to wait for future analysis and consider the position of a treatment method that combines atezolizumab and bevacizumab for HAIC for patients with advanced HCC with Vp4. A subgroup analysis in the SILIUS trial of patients with $\mathrm{Vp} 4$ showed that those treated with HAIC combined with sorafenib had a better prognosis compared with those treated with sorafenib alone. Therefore, similarly, it is expected that the combined use of atezolizumab and bevacizumab in HAIC may further improve the overall survival.

There are many reports that HAIC is an effective treatment for advanced HCC with PVTT. Some retrospective studies that compared sorafenib with HAIC for advanced HCC with PVTT showed a better OS after HAIC than after sorafenib $[22,23,29]$. The presence or absence of MVI was not identified as a prognostic factor in HAIC-treated patients with advanced HCC in those reports.

The SILIUS trial was conducted as a prospective study of HAIC for patients with advanced HCC, comparing sorafenib plus HAIC using low-dose FP versus sorafenib alone. There was no difference in prognosis between the 2 treatment arms overall. However, a subgroup analysis of patients with advanced HCC with $\mathrm{Vp} 4$ showed that sorafenib plus HAIC resulted in a better prognosis (median OS = 11.4 months) compared with sorafenib alone (median OS $=6.5$ months) [25]. Ikeda et al. [26] compared sorafenib plus HAIC using CDDP versus sorafenib alone for advanced HCC patients with PVTT ranging from Vp1 to Vp4, of whom 17 and 40 were randomly assigned to the 2 treatment arms, respectively. The prognosis was better in the sorafenib plus HAIC group (median $\mathrm{OS}=10.6$ months) than in the sorafenib alone group (median OS $=8.7$ months). This is a phase II trial, so it is hard to say that HAIC has been established as a standard treatment for advanced HCC. However, HAIC might be considered an effective treatment for advanced HCC with PVTT. There are 2 HAIC regimens, both of which showed better results compared with sorafenib in previous trials comparing HAIC with sorafenib.

In addition, recently, the results of 2 randomized controlled trials have been published from China and Korea. The first trial was a phase III trial of sorafenib plus HAIC with FOLFOX versus sorafenib for patients with advanced HCC with PVTT. The overall survival and PFS 
were significantly longer in sorafenib plus HAIC with FOLFOX than in sorafenib alone [33]. The other trial was a randomized controlled trial of sorafenib versus HAIC with 5-FU plus CDDP for patients with advanced HCC with $\mathrm{Vp} 3$ or 4 . The overall survival and time to progression were significantly longer in HAIC with 5-FU plus CDDP than in sorafenib alone [34]. As a result, HAIC has become to attract attention again.

Recent advances in RT technology have established its efficacy and safety. Favorable results of combination HAIC and RT for advanced HCC with MVI have been reported. In those reports, the median OS and ORR of patients with PVTT were 8.6-13.1 months and 45-56\%, respectively $[7,28,29,35]$. RT appeared to have reduced the PVTT volume, increased blood flow to the liver parenchyma, improved the hepatic functional reserve, and decreased portal hypertension-related events. Therefore, we consider that the addition of RT to HAIC for HCC patients with PVTT is useful. However, it remains unclear regarding the OS, PFS, response rate, and safety of the combination therapy of HAIC and RT in HCC patients with Vp4 in previous reports. However, those reports involved advanced HCC with PVTT ranging from Vp1 to $\mathrm{Vp} 4$, with no studies conducted on $\mathrm{Vp} 4$ cases alone; thus, there is no established treatment for advanced HCC with Vp4. Although, the effectiveness of additional radiotherapy remained to be clarified, in a 2015 paper, Fujino et al. [28] reported the objective response of PVTT was significantly higher in the RT group (56.1\%) than in the nonRT group (33.3\%), while that of intrahepatic tumor and OS were not significantly different between groups. Median OS, time-to-treatment failure, and post-progression survival were significantly longer in the RT group than in the non-RT group (8.6 and 5.0 months, 5.0 and 2.7 months, and 5.3 and 1.5 months, respectively) among intrahepatic tumor nonresponders to HAIC. By multivariate analysis, the combination of 3D-CRT with HAIC was an independent contributing factor for OS (HR, 3.2; 95\% confidence interval, 1.692-6.021; $p<0.001)$ among intrahepatic HCC nonresponders to HAIC. Therefore, we think that additional therapy of TACE or TKI can be continued as a result of the increased blood flow in the liver parenchyma and retention of hepatic functional reserve achieved by RT among intrahepatic HCC nonresponders to HAIC [28].

The subject in this study was limited to HCC patients with Vp4 cases. The ORR of patients with PVTT was $51.0 \%$, and the median OS of the responders was 19.4 months. Our results were better than those of previous studies. As a retrospective single-arm study with a small sample size, there were some limitations. The HAIC regimen contains a variety of drugs and the irradiation dose was around 30 or $45 \mathrm{~Gy}$. We think that it is necessary to increase the number of cases and analyze the difference depending on the regimen of HAIC and RT. However, the combination therapy of HAIC and RT was well tolerated and did not develop RILD in all cases. We believe that the combination therapy of HAIC and RT for HCC patients with Vp4 may be a new therapy for future analysis, and our results warrant further studies.

\section{Acknowledgements}

Not applicable.

\section{Statement of Ethics}

This study was conducted in accordance with the ethical principles of the Declaration of Helsinki and was approved by the Institutional Review Board of Hiroshima University (IRB number 695). Written informed consent was obtained from each patient after a detailed explanation of the study procedures.

\section{Conflict of Interest Statement}

The authors declare that they have no conflict of interest.

\section{Funding Sources}

This work was not supported.

\section{Author Contributions}

Y. Kosaka and T. Kawaoka designed the study, analyzed the data, and wrote the paper; Y. Kosaka acquired the data; Y. Kosaka performed the statistical analysis; T. Kawaoka and H. Aikata reviewed the results; $\mathrm{H}$. Aikata revised the manuscript for important intellectual content.

References

1 Forner A, Reig M, Bruix J. Hepatocellular carcinoma. Lancet. 2018;391(10127):1301-14.

2 Jemal A, Bray F, Center MM, Ferlay J, Ward E, Forman D. Global cancer statistics. CA Cancer J Clin. 2011;61(2):69-90.

3 Ando E, Yamashita F, Tanaka M, Tanikawa K. A novel chemotherapy for advanced hepatocellular carcinoma with tumor thrombosis of the main trunk of the portal vein. Cancer. 1997;79(10):1890-6. 
4 Rossi S, Di Stasi M, Buscarini E, Quaretti P, Garbagnati F, Squassante L, et al. Percutaneous RF interstitial thermal ablation in the treatment of hepatic cancer. AJR Am J Roentgenol. 1996;167(3):759-68.

5 Llovet JM, Real MI, Montaña X, Planas R, Coll $S$, Aponte J, et al. Arterial embolisation or chemoembolisation versus symptomatic treatment in patients with unresectable hepatocellular carcinoma: a randomised controlled trial. Lancet. 2002 May 18;359(9319): 1734-9.

6 Jang BK, Chung WJ, Park KS, Cho KB, Hwang JS, Ahn SH, et al. [The efficacy of hepatic arterial infusion therapy for advanced hepatocellular carcinoma according to extrahepatic collateral feeding vessels]. Korean J Hepatol. 2005 Dec;11(4):359-70.

7 Kim DY, Park W, Lim DH, Lee JH, Yoo BC, Paik SW, et al. Three-dimensional conformal radiotherapy for portal vein thrombosis of hepatocellular carcinoma. Cancer. 2005 Jun 1; 103(11):2419-26.

8 Obi S, Yoshida H, Toune R, Unuma T, Kanda $\mathrm{M}$, Sato S, et al. Combination therapy of intraarterial 5-fluorouracil and systemic interferon-alpha for advanced hepatocellular carcinoma with portal venous invasion. Cancer. 2006 May 1;106(9):1990-7.

9 Uka K, Aikata H, Takaki S, Miki D, Kawaoka T, Jeong SC, et al. Pretreatment predictor of response, time to progression, and survival to intraarterial 5-fluorouracil/interferon combination therapy in patients with advanced hepatocellular carcinoma. J Gastroenterol. 2007 Oct;42(10):845-53.

10 Llovet JM, Ricci S, Mazzaferro V, Hilgard P, Gane E, Blanc JF, et al. Sorafenib in advanced hepatocellular carcinoma. N Engl J Med. 2008 Jul 24;359(4):378-90.

11 Cheng AL, Kang YK, Chen Z, Tsao CJ, Qin S, Kim JS, et al. Efficacy and safety of sorafenib in patients in the Asia-Pacific region with advanced hepatocellular carcinoma: a phase III randomised, double-blind, placebo-controlled trial. Lancet Oncol. 2009 Jan; 10(1): 25-34.

12 Kim HY, Kim JD, Bae SH, Park JY, Han KH, Woo HY, et al. A comparative study of highdose hepatic arterial infusion chemotherapy and transarterial chemoembolization using doxorubicin for intractable, advanced hepatocellular carcinoma. Korean J Hepatol. 2010 Dec;16(4):355-61.

13 Ueshima K, Kudo M, Takita M, Nagai T, Tatsumi C, Ueda T, et al. Hepatic arterial infusion chemotherapy using low-dose 5-fluorouracil and cisplatin for advanced hepatocellular carcinoma. Oncology. 2010 Jul;78(Suppl 1):14853.

14 Takayasu K, Arii S, Kudo M, Ichida T, Matsui O, Izumi N, et al. Superselective transarterial chemoembolization for hepatocellular carcinoma. Validation of treatment algorithm proposed by Japanese guidelines. J Hepatol. 2012 Apr;56(4):886-92.
15 Hasegawa K, Kokudo N, Makuuchi M, Izumi N, Ichida T, Kudo M, et al. Comparison of resection and ablation for hepatocellular carcinoma: a cohort study based on a Japanese nationwide survey. J Hepatol. 2013 Apr;58(4): 724-9.

16 Bruix J, Qin S, Merle P, Granito A, Huang YH, Bodoky G, et al. Regorafenib for patients with hepatocellular carcinoma who progressed on sorafenib treatment (RESORCE): a randomised, double-blind, placebo-controlled, phase 3 trial. Lancet. 2017 Jan 7;389(10064): 56-66.

17 Kudo M, Finn RS, Qin S, Han KH, Ikeda K, Piscaglia F, et al. Lenvatinib versus sorafenib in first-line treatment of patients with unresectable hepatocellular carcinoma: a randomised phase 3 non-inferiority trial. Lancet. 2018 Mar 24;391(10126):1163-73.

18 Personeni N, Rimassa L, Pressiani T, Smiroldo V, Santoro A. Cabozantinib for the treatment of hepatocellular carcinoma. Expert Rev Anticancer Ther. 2019 Oct;19(10):847-55.

19 Zhu AX, Kang YK, Yen CJ, Finn RS, Galle PR, Llovet JM, et al. Ramucirumab after sorafenib in patients with advanced hepatocellular carcinoma and increased alpha-fetoprotein concentrations (REACH-2): a randomised, double-blind, placebo-controlled, phase 3 trial. Lancet Oncol. 2019 Feb;20(2):282-96.

20 Fujii T, Takayasu K, Muramatsu Y, Moriyama N, Wakao F, Kosuge T, et al. Hepatocellular carcinoma with portal tumor thrombus: analysis of factors determining prognosis. Jpn J Clin Oncol. 1993 Apr;23(2):105-9.

21 Ando E, Tanaka M, Yamashita F, Kuromatsu R, Yutani S, Fukumori K, et al. Hepatic arterial infusion chemotherapy for advanced hepatocellular carcinoma with portal vein tumor thrombosis: analysis of 48 cases. Cancer. 2002 Aug 1;95(3):588-95.

22 Song DS, Song MJ, Bae SH, Chung WJ, Jang JY, Kim YS, et al. A comparative study between sorafenib and hepatic arterial infusion chemotherapy for advanced hepatocellular carcinoma with portal vein tumor thrombosis. J Gastroenterol. 2015 Apr;50(4):445-54.

23 Kawaoka T, Aikata H, Kobayashi T, Uchikawa S, Ohya K, Kodama K, et al. Comparison of hepatic arterial infusion chemotherapy between 5-fluorouracil-based continuous infusion chemotherapy and low-dose cisplatin monotherapy for advanced hepatocellular carcinoma. Hepatol Res. 2018 Dec;48(13):1118-30.

24 Court WS, Order SE, Siegel JA, Johnson E, DeNittis AS, Principato R, et al. Remission and survival following monthly intraarterial cisplatinum in nonresectable hepatoma. Cancer Invest. 2002;20(5-6):613-25.

25 Kudo M, Ueshima K, Yokosuka O, Ogasawara S, Obi S, Izumi N, et al. Sorafenib plus low-dose cisplatin and fluorouracil hepatic arterial infusion chemotherapy versus sorafenib alone in patients with advanced hepatocellular carcinoma (SILIUS): a randomised, open label, phase 3 trial. Lancet Gastroenterol Hepatol. 2018 Jun;3(6):424-32.
26 Ikeda M, Shimizu S, Sato T, Morimoto M, Kojima Y, Inaba Y, et al. Sorafenib plus hepatic arterial infusion chemotherapy with cisplatin versus sorafenib for advanced hepatocellular carcinoma: randomized phase II trial. Ann Oncol. 2016 Nov;27(11):2090-6.

27 Toya R, Murakami R, Baba Y, Nishimura R, Morishita S, Ikeda O, et al. Conformal radiation therapy for portal vein tumor thrombosis of hepatocellular carcinoma. Radiother Oncol. 2007 Sep;84(3):266-71.

28 Fujino H, Kimura T, Aikata H, Miyaki D, Kawaoka T, Kan H, et al. Role of 3-D conformal radiotherapy for major portal vein tumor thrombosis combined with hepatic arterial infusion chemotherapy for advanced hepatocellular carcinoma. Hepatol Res. 2015 Jun; 45(6):607-17.

29 Kodama K, Kawaoka T, Aikata H, Uchikawa S, Nishida Y, Inagaki Y, et al. Comparison of outcome of hepatic arterial infusion chemotherapy combined with radiotherapy and sorafenib for advanced hepatocellular carcinoma patients with major portal vein tumor thrombosis. Oncology. 2018;94(4):215-22.

30 Eisenhauer EA, Therasse P, Bogaerts J, Schwartz LH, Sargent D, Ford R, et al. New response evaluation criteria in solid tumours: revised RECIST guideline (version 1.1). Eur J Cancer. 2009 Jan;45(2):228-47.

31 Arnold B, Mitchell SA, Lent L, Mendoza TR, Rogak LJ, Barragan NM, et al. Linguistic validation of the Spanish version of the National Cancer Institute's patient-reported outcomes version of the common terminology criteria for adverse events (PRO-CTCAE). Support Care Cancer. 2016 Jul;24(7):2843-51.

32 Finn RS, Qin S, Ikeda M, Galle PR, Ducreux M, Kim TY, et al. Atezolizumab plus bevacizumab in unresectable hepatocellular carcinoma. N Engl J Med. 2020 May 14;382(20): 1894-905.

33 He M, Li Q, Zou R, Shen J, Fang W, Tan G, et al. Sorafenib plus hepatic arterial infusion of oxaliplatin, fluorouracil, and leucovorin vs. sorafenib alone for hepatocellular carcinoma with portal vein invasion: a randomized clinical trial. JAMA Oncol. 2019;5(7):953-60.

34 Choi JH, Chung WJ, Bae SH, Song DS, Song MJ, Kim YS, et al. Randomized, prospective, comparative study on the effects and safety of sorafenib vs. hepatic arterial infusion chemotherapy in patients with advanced hepatocellular carcinoma with portal vein tumor thrombosis. Cancer Chemother Pharmacol. 2018;82(3):469-78.

35 Han KH, Seong J, Kim JK, Ahn SH, Lee DY, Chon CY. Pilot clinical trial of localized concurrent chemoradiation therapy for locally advanced hepatocellular carcinoma with portal vein thrombosis. Cancer. 2008;113(5): 995-1003. 